Cahiers de Narratologie

\title{
La triple tension narrative : chrono-topique, pathétique, télique
}

\section{Gabriel Sevilla}

\section{(2) OpenEdition \\ Journals}

Electronic version

URL: http://journals.openedition.org/narratologie/6901

DOI: 10.4000/narratologie.6901

ISSN: 1765-307X

Publisher

LIRCES

Electronic reference

Gabriel Sevilla, "La triple tension narrative : chrono-topique, pathétique, télique », Cahiers de Narratologie [Online], 26 | 2014, Online since 17 September 2014, connection on 05 May 2019. URL http://journals.openedition.org/narratologie/6901; DOI : 10.4000/narratologie.6901

This text was automatically generated on 5 May 2019.

Article L.111-1 du Code de la propriété intellectuelle. 


\title{
La triple tension narrative : chrono- topique, pathétique, télique
}

\author{
Gabriel Sevilla
}

1 Le concept de «tension narrative » est devenu, pour la narratologie contemporaine, une reformulation implicite de ce qui est probablement la question la plus ancienne et la plus fondamentale de la discipline. Il s'agirait du récit lui-même comme objet d'étude, des traits qui le distinguent d'autres actes communicatifs et cognitifs, et non plus seulement des sous-catégories narratives et de leurs problématiques particulières. La tension narrative nous renvoie ainsi à un vieux secret de Polichinelle : de l'Antiquité à nos jours, on n'est pas parvenu à un vrai consensus sur la définition de l'acte de raconter, qu'on parle de récit, de narrativité, etc. (Abbott 2011) adoptant une approche ou une autre (Nünning 2003; Meister 2013). Certes, on a dressé de multiples histoires du récit, de multiples théories du récit, des histoires des théories du récit, etc., mais un accord plus ou moins large sur la définition de l'objet d'étude lui-même resterait toujours à atteindre. La première vertu de la tension narrative serait ainsi d'avoir reformulé la question qui pointe le vide majeur et le plus ancien de la discipline.

2 Une deuxième vertu de la tension serait d'avoir creusé la dimension cognitive du récit, délivrant la perspective du récepteur de l'objectivisme que les poétiques de la réception avaient hérité silencieusement du structuralisme. La définition du concept par son auteur, Raphaël Baroni, en est probablement la meilleure illustration (2007: 18) : «la tension est le phénomène qui survient lorsque l'interprète d'un récit est encouragé à attendre un dénouement, cette attente étant caractérisée par une anticipation teintée d'incertitude qui confère des traits passionnels à l'acte de réception». Baroni nous propose une définition relationnelle du récit comme interaction effective d'un récepteur dans la communication. Ce récepteur ne serait plus le simple évaluateur final d'un objet aux traits supposément "descriptibles en soi », tel que le posaient implicitement des théoriciens de la réception comme Umberto Eco (1985a : 137) : « une narration est une description d'actions qui requiert pour chaque action décrite un agent $[. .]$.$» , etc. { }^{1}$. Le récepteur serait, du point de vue de la tension, le critère même de la définition du récit, ce qui nous obligerait à rapporter constamment les traits communicatifs de la narrativité 
aux mécanismes cognitifs qui les actualisent. Nous ne devrions donc plus nous interroger sur le récit, mais sur l'acte de lecture narrative ou, plus largement, sur l'acte de communication (et de cognition) narrative. Le récepteur transformerait ainsi la vieille notion de récit dans un type de réponse que nous appellerions tension, et la psychologie cognitive s'avérerait une précieuse nomenclature complémentaire à celles de la linguistique, de la rhétorique et, plus largement, de la sémiotique.

Nous essayerons d'approfondir cette perspective à travers deux hypothèses. La première entend qu'on pourrait encore reformuler la question du récit, nuançant quelques-uns de ses aspects fondamentaux, à travers une structuration interne du concept de Baroni en trois volets: tension chrono-topique, tension pathétique et tension télique. Nous expliquerons ces trois sous-tensions de la tension narrative par des éléments linguistiques, psychologiques, rhétoriques, etc., d'un point de vue autant communicatif que cognitif. L'accent sera pourtant mis sur ce dernier, à notre avis indispensable au récit, ce qui nous amènera à nuancer la théorie de Baroni à ce sujet. Pour ainsi dire, notre première hypothèse proposera non seulement une tripartition de la tension narrative, mais aussi sa pleine orientation cognitive ${ }^{2}$.

4 Notre deuxième hypothèse affirme qu'on pourrait distinguer les récits verbaux et iconiques, d'une part, des récits musicaux, d'autre part, en définissant les premiers par les trois tensions susmentionnées et les deuxièmes par une articulation particulière de la tension télique seulement. Qu'on parle de récit ou de proto-récit musical ${ }^{3}$, ce qui nous paraît fondamental c'est de souligner comment la musique réussit à articuler, par ses propres unités culturelles, la tension télique d'un suspense (Grabócz 2011 : 11-12) à l'écart des tensions chrono-topique et pathétique. Le suspense étant une notion qu'on ne saurait pas trouver ailleurs que dans le récit, contrairement aux notions de chrono-tope et de pathos, nous serions enclins à garder une certaine association (l'étiquette étant secondaire) entre les notions de suspense musical et de récit ou narrativité. Le suspense musical pourrait devenir, par ailleurs, d'une grande importance pour l'étude cognitive du suspense iconique et verbal, dans la mesure où il détache l'activité mentale qui lui est propre des ancrages plus concrets de l'espace-temps et de la sensibilité des personnages. La musique nous permettrait, pour ainsi dire, d'envisager l'acte cognitif narratif comme un suspense "dans l'abstrait», réduit à son articulation temporelle de souvenirs et d'attentes, ce qui pourrait défricher la voie des hypothèses cognitives les plus audacieuses, notamment celles qui identifient l'acte de raconter à l'acte même de penser.

Cet article sera consacré dans son intégralité au développement théorique de nos deux hypothèses à travers la définition des trois tensions susmentionnées. L'ensemble de notre proposition vise, cela va sans dire, à l'analyse textuelle qui lui accorde un plein sens et qui lui permet d'avancer dans le débat auquel elle essaie de contribuer. Nous développons cette analyse ailleurs, nous penchant ici sur la stricte définition des concepts ${ }^{4}$. Comme conclusion de celle-ci, nous proposerons deux réponses possibles à la vieille question du récit du point de vue de la (triple) tension narrative, c'est-à-dire deux approches sur l'objet d'étude de la narratologie comme acte communicatif et cognitif inter-linguistique.

\section{Tension chrono-topique ${ }^{5}$}

6 On pourrait la définir comme l'immersion cognitive de l'émetteur et/ou le récepteur dans l'espace-temps défini et cohérent d'une unité culturelle ${ }^{6}$, qu'elle soit narrative ou non, qu'elle ait un référent ou non, que ce dernier (s'il existe) soit présent ou absent. Cette 
immersion se produirait au point de nous «faire oublier " momentanément l'espacetemps de notre réception: salle de cinéma, bibliothèque, etc. Sous de différentes étiquettes, ce phénomène a été étudié de très différentes approches, allant de la linguistique pragmatique (Karl Bühler) à la théorie filmique (Noël Burch), en passant par la psychanalyse filmique (Christian Metz), la théorie littéraire des mondes possibles (David Lewis, Thomas Pavel, Umberto Eco, etc.), l'intelligence artificielle (Stuart C. Shapiro, Janyce M. Wiebe, etc.) et autres (Duchan et al. : 1995). Pour expliquer ses traits communicatifs et cognitifs fondamentaux, nous ferons appel à la linguistique pragmatique en lien avec quelques notions de théorie filmique. La pragmatique nous fournit un système descriptif très précis, mettant en valeur la base iconique de la deixis verbale et introduisant une perspective cognitive élémentaire. La théorie filmique développe cette base iconique et approfondit sa dimension cognitive. La musique n'aura pas de place dans ce schéma. Certes, elle a sa propre spatialité et temporalité du point de vue communicatif et cognitif, mais l'éventuelle substitution de celles-ci à l'espace-temps de la réception se fait de manière beaucoup plus faible et irrégulière qu'en parole et image.

\section{Approche pragmatique}

7 Bühler définit l'immersion spatio-temporelle du récepteur dans l'allusion verbale à un référent, qu'il soit fictif ou absent, par la notion de Deixis am Phantasma, traduite en français comme "deixis à l'imaginaire » (Bühler 2009: passim). Celle-ci part du concept traditionnel de deixis comme demonstratio ad oculos, c'est-à-dire comme l'acte communicatif par lequel un émetteur guide son récepteur auprès d'un référent qui existe, qui est présent et que le récepteur voit de ses propres yeux. Dans son guidage, l'émetteur peut faire appel aux auxiliaires déictiques pré-linguistiques («la flèche que forment le bras tendu et l'index d'un locuteur »), ainsi qu'aux adverbes de temps et de lieu, aux pronoms personnels, aux démonstratifs, etc. (Bühler 2009: 230). Tous ces éléments l'aident à récréer verbalement l'espace-temps d'une situation communicative partagée avec le récepteur. La demonstratio ad oculos est ainsi une sorte de deixis in praesentia ou in situ.

8 Par opposition, la Deixis am Phantasma est une deixis fictive ou in absentia : le récepteur et, éventuellement, l'émetteur ne se trouvent pas auprès de la réalité évoquée par les mots, soit parce qu'elle n'existe pas (imagination), soit parce qu'elle est ailleurs (mémoire). Ainsi, la Deixis am Phantasma se produit (Ibid.) :

[...] lorsqu'un narrateur guide un auditeur dans le royaume de ce qui est absent et accessible par le souvenir, voire dans le royaume de l'imagination constructive, et qu'il le traite là avec les mêmes termes déictiques [que dans une demonstratio ad oculos], de façon à ce qu'il voie en entende ce qu'il y a là à voir et à entendre (et à toucher, cela va de soi, et peut-être même à sentir et à goûter).

Cette notion pourrait s'appliquer à tout acte communicatif, qu'il soit narratif ou non. Il est cependant très éloquent que Bühler parle d'un "narrateur " et non pas d'un " émetteur ", nous renvoyant ainsi au récit comme domaine par excellence de la Deixis am Phantasma. On pourrait ranger celle-ci, ainsi que la demonstratio ad oculos qui est à sa base, dans ce que nous avons appelé tension chrono-topique. Certes, la définition de Bühler se focalise sur le domaine verbal et notamment sur son versant oral, puisqu'il parle d'un " auditeur ». Rien ne nous empêche, pourtant, d'extrapoler ces notions au domaine iconique, où des deixis ad oculos et am Phantasma se développent parfaitement en absence 
de mots, comme le montrent les exemples de la pantomime, de la peinture figurative ou du cinéma ${ }^{7}$. À plus forte raison si l'on considère que le latin oculos (yeux), ainsi que l'allemand Phantasma (vision), nous renvoient tous les deux étymologiquement au domaine du visuel-iconique comme base de la deixis ${ }^{8}$. En effet, partant d'une image physique (ad oculos) et s'adressant à une image mentale (am Phantasma), la notion de deixis a été pourtant ramenée au verbal, c'est-à-dire à l'éventuel pont non-iconique entre les deux. C'est avec l'invention du cinéma qu'une théorie proprement iconique de la deixis s'est développée du point de vue cognitif ébauché par Bühler. L'importance de la théorie filmique vis-à-vis de la tension chrono-topique est justement celle-ci : d'avoir renversé son logocentrisme en même temps qu'elle développait son approche cognitive. Logocentrisme qu'on peut retracer dans sa plus grande contradiction chez Bühler luimême, capable de construire sa réflexion sur les notions ad oculos et am Phantasma sans pour autant remettre en question la centralité déictique de la parole.

\section{Deux critères de classement}

Dans la communication, les tensions chrono-topiques verbale et iconique s'articulent de manières antithétiques. Nous nous en tiendrons ici aux deux antithèses fondamentales. La première porte sur l'opposition même du verbal à l'iconique, sur la nature essentiellement différente de leurs mimesis ou, si l'on veut, sur les différents rapports que leurs tensions chrono-topiques entretiennent vis-à-vis de notre expérience sensible. Cette première différence, qu'on pourrait appeler sémantique, se traduit par la dichotomie : analogique vs. non-analogique ${ }^{9}$. Les images figuratives ont, par leur mimesis analogique (soit de notre expérience sensible, soit d'une prolongation fictive de celle-ci), la capacité de construire une deixis in absentia (tableau, film, etc.) formellement semblable à ses possibles équivalents in praesentia (paysage, plateau, etc.). Les mots, en revanche, par leur mimesis non-analogique (soit de notre expérience sensible, soit d'une prolongation fictive de celle-ci), construisent toujours une deixis in absentia (conversation, roman, etc.) formellement différente de ses possibles équivalents in praesentia (situation vécue, faits quotidiens, etc.). Les mimesis analogique et nonanalogique nous renvoient toujours, qu'elles aient un référent ou non, à des unités culturelles. Dans le cadre du récit, de telles unités véhiculent non seulement les coordonnées d'espace-temps, mais aussi les connaissances encyclopédiques plus larges que l'émetteur et/ou le récepteur peut projeter sur cet espace-temps. La première et plus importante différence au sein de ces unités résiderait dans le caractère analogique (iconique) ou non-analogique (verbal) de leur mimesis.

11 La deuxième opposition, cette fois-ci transversale aux langages verbal et iconique, porte sur l'intensité perceptive (physique-cognitive) de la tension chrono-topique. L'opposition pertinente ici, qu'on pourrait appeler pragmatique, est: dynamique vs. non-dynamique. Dans le domaine verbal, il est évident que la communication orale impose un rythme de réception (écoute) qui n'est normalement pas choisi par le récepteur, alors que la communication écrite est en général soumise au rythme de réception (lecture) librement choisi par le récepteur. Le signe verbal oral «bouge », pour ainsi dire, alors que le signe verbal écrit «est fait bouger ». Pour un même récit verbal nous parlerions, de la sorte, d'une tension chrono-topique a priori plus intense dans sa version orale (dynamique) que dans sa version écrite (non-dynamique). De manière analogue nous avons, au domaine iconique, une opposition entre l'image fixe (non-dynamique) et l'image en mouvement 
(dynamique). Face à une même suite d'images, la version dynamique atteindrait raisonnablement une tension chrono-topique plus élevée que la version non-dynamique. Si nous considérons, enfin, la mimesis analogique des images comme étant plus intense (moins abstraite) sur le plan perceptif que la mimesis non-analogique des mots, nous pouvons classer les différentes tensions chrono-topiques du minimum au maximum degré d'intensité : signe non-analogique et non-dynamique (mot écrit); signe analogique et non-dynamique (peinture, photographie, etc.); signe non-analogique et dynamique (parole, sous-titres); signe analogique et dynamique (cinéma, théâtre, danse, etc.). L'entrelacement ici du verbal et de l'iconique, nettement séparés avant par le critère d'analogie, met encore une fois en évidence la portée inter-linguistique d'une notion d'espace-temps traditionnellement ramenée à sa perspective verbale.

\section{Théorie filmique}

Le cinéma appelle ici un commentaire à part. Par son intensité chrono-topique superlative, il a poussé très tôt ses théoriciens à développer l'approche pragmatique qui garantissait sa spécificité comme langage. Du point de vue chrono-topique, en effet, la principale différence entre le cinéma et les autres mimesis analogiques fixées sur un support (photographie, peinture, etc.) est strictement pragmatique : dynamique vs. nondynamique. C'est au matérialisme historique qu'on doit l'une des plus précoces et fécondes remarques pragmatiques sur le cinéma, c'est-à-dire sur l'impact perceptif de l'image en mouvement. Face aux premiers films, d'une intensité chrono-topique historiquement inouie, Walter Benjamin est allé jusqu'à assimiler leur effet sur le public au «choc physique» des avant-gardes artistiques de l'époque: «Par sa technique, le cinéma a délivré l'effet de choc physique de la gangue morale où le dadaïsme l'avait en quelque sorte enfermé » (2006: 68-69). Non sans lien aux positions matérialistes, Noël Burch s'est référé plus récemment à l'impression de réalité au cinéma d'Hollywood comme "un sentiment plein et entier d'être-là » auquel nous serions induits par la « création d'un espace habitable » à travers le montage (1991: 250). Ce « sentiment d'être là » reviendrait au processus cognitif d'immersion spatio-temporelle qu'on a appelée ici tension chrono-topique. L'intérêt de l'approche de Burch serait d'avoir développé sur le plan filmique, mais au niveau strictement cognitif, la tension chrono-topique que Benjamin assimilait pragmatiquement à un «choc». On dirait qu'un tel «choc» a été nécessaire pour révéler l'impact physique-cognitif de toute deixis, iconique ou verbale, ce qui a mené au développement de son «impact » spécifiquement cognitif : l'immersion spatio-temporelle.

13 Aujourd'hui, les tests neurologiques faisant appel au récit pour expliquer les phénomènes d'immersion sont largement plus basés sur des exemples filmiques que littéraires (Keen 2013 : 11). Ce tournant iconique dans l'étude de la représentation spatio-temporelle nous permet de repenser l'ensemble du système déictique, du sommet à la base, et de contourner les pièges de son logocentrisme, ainsi que de combler ses lacunes cognitives. $\mathrm{Au}$ sommet du système, la tension chrono-topique du cinéma met en évidence par son degré superlatif les aspects sémantiques et pragmatiques de toute tension chronotopique, iconique ou verbale, comme immersion cognitive spatio-temporelle. À la lumière $\mathrm{du}$ "choc physique" nous pourrions expliquer un roman et un film comme deux manières antithétiques, non-analogique et non-dynamique (roman), analogique et dynamique (film), de susciter chez nous « un sentiment d'être-là ». À la base du système 
on a ce que Bühler appelait les auxiliaires déictiques pré-linguistiques : «la flèche que forment le bras tendu et l'index d'un locuteur». Ces éléments iconiques ne seraient souvent pas auxiliaires ni bien entendu antérieurs à la notion de langage, mais un langage en eux-mêmes et fréquemment autonome, le langage verbal pouvant même devenir auxiliaire à leur égard. Ainsi, aux niveaux plus simple et plus complexe de la deixis, à la base et au sommet de son système conceptuel nous trouverions surtout des images. On pourrait même dire que le cinéma et la gestualité encadrent iconiquement la notion verbale de deixis. Si on accepte cette position privilégiée de l'image pour expliquer les processus d'immersion spatio-temporelle, sa nature plus intense et explicite comme objet d'étude cognitive, on devrait peut-être repenser notre nomenclature d'un point de vue iconique. Les deux dichotomies, sémantique et pragmatique, proposées plus haut essaient d'avancer dans cette direction.

\section{Tension pathétique}

On pourrait la définir comme l'immersion cognitive de l'émetteur et/ou le récepteur dans le pathos (émotions et sensations ${ }^{10}$ ) d'un autre, symbolique ou réel, en adoptant sa perspective. Cela nous amène à un deuxième processus cognitif qui est simultané du premier: la construction du récit comme dimension éthique. On devrait comprendre cette construction au sens large, c'est-à-dire comme la capacité de l'émetteur et/ou le récepteur à articuler des jugements sur la dichotomie bien/mal dans quelque sens que ce soit, et non pas comme la projection d'une moralité quelconque. La tension pathétique se montre ainsi comme une immersion cognitive spécialement complexe. Pour expliquer ses traits communicatifs et cognitifs fondamentaux nous utiliserons quelques références contemporaines, autant narratologiques qu'en neurosciences, sur l'immersion cognitive de l'empathie : modalité plus intense, mais pas exclusive, de la tension pathétique.

point de vue des neurosciences cognitives, des auteurs comme Simon Baron-Cohen assimilent l'empathie à "notre capacité d'identifier ce que quelqu'un d'autre pense ou sent et de répondre à ses pensées et à ses sentiments par une émotion appropriée ${ }^{11}$ " (2011 : 16). Une certaine idée de tension sous-tendrait cette réaction obligée au stimulus de départ: «il y a du moins deux étapes dans l'empathie : reconnaissance et réponse » $\left(\mathrm{Ibid}^{12}\right.$.). En absence de reconnaissance et/ou de réponse, on aboutirait dans un manque d'empathie qui pourrait aller de l'extrême zéro-négatif (psychopathe) à l'extrême zéropositif (Asperger) mais qui serait toujours lié, d'après Baron-Cohen et autres, aux aptitudes éthiques des individus ${ }^{13}$. Ce qui est fondamental pour nous ici c'est que, afin de vérifier ces hypothèses, les neurosciences ont fait appel à la réception narrative comme une de leurs pierres de touche : «Nombre des premières études en lecture de pensée ou empathie chez des gens zéro-positifs se fondaient sur des tests verbaux (par exemple, interpréter des récits ou des commentaires sarcastiques ou classer des émotions) » $\left(2011: 101^{14}\right)$.

16 Du point de vue de la narratologie cognitive, qui a considérablement puisé aux sources des neurosciences et de la psychologie, des auteurs comme Suzanne Keen définissent l'empathie comme «le partage d'une sensibilité et l'adoption d'une perspective induits par la lecture, le visionnement, l'écoute ou l'imagination des récits de la situation et de la condition d'autrui » $(2013: 2)^{15}$. Keen se focalise sur la «sensibilité partagée » et la " perspective adoptée » par un émetteur et/ou un récepteur vis-à-vis d'un personnage, creusant ainsi les aspects poétiques et rhétoriques liés aux processus cognitifs que les 
neurosciences étudient strictement en termes d'activité cérébrale. Substituant la notion de pathos à celle de sensibilité, nous nous baserons ici sur les mêmes deux piliers que Keen pour développer le concept de tension pathétique.

\section{Adoption d'une perspective}

17 Les récits verbaux comptent avec le système de la deixis personnelle (première, seconde et troisième personne du singulier ou du pluriel) pour articuler l'adoption d'une perspective. Ce système a par ailleurs la vertu de nous permettre aborder dans un même schéma les tensions pathétique (deixis personnelle) et chrono-topique (deixis spatiotemporelle). Parallèlement, il nous faut aussi une déclination des personnes de la « conjugaison iconique » et, éventuellement, des personnes de la " conjugaison sonore » afin de déterminer les différentes perspectives possibles au récit. Pour les récits iconiques on empruntera les notions d'ocularisation (image) et d'auricularisation (image-son) de François Jost (1989).

18 Dans le domaine iconique, Jost appelle ocularisation zéro le point de vue qui ne s'identifie à "aucun œil interne à la diégèse", c'est-à-dire à aucun personnage (1989: 26). L'ocularisation zéro reviendrait à un narrateur qui ne se présenterait pas comme tel, qu'il soit omniscient, behavioriste ou narrateur-personnage. On pourrait penser à une équivalence avec la troisième personne de la conjugaison verbale, qui s'avérerait singulière ou plurielle en fonction de la qualité simple ou complexe de son objet. Plus concrètement, la notion iconique de «zéro » fonctionnerait comme synonyme implicite de la notion verbale d'impersonnel: une troisième personne sans identité définie. Par opposition à celle-ci, Jost appelle ocularisation interne le point de vue appartenant à un personnage présenté comme tel, où il distingue deux variantes : primaire et secondaire. Dans les deux cas nous aurons affaire à une première personne du singulier. L'ocularisation interne secondaire, la plus commune, serait une première personne construite " par le montage, les raccords (comme dans le champ-contrechamp) ou par le verbal (cas d'une accroche dialoguée), en bref, par une contextualisation » (Ibid.). Jost donne l'exemple du raccord de regard comme ocularisation interne secondaire par excellence (Ibid. : 29) : «n'importe quelle image succédant à un plan mettant en scène un personnage qui regarde sera sentie comme appartenant à son paysage visuel ». L'ocularisation interne primaire, quant à elle, serait une première personne "où se marque dans le signifiant [l'image] la matérialité d'un corps ou la présence d'un œil qui permet immédiatement, sans le secours du contexte [montage], d'identifier un personnage absent de l'image » (1989: 27). Ce serait le cas, par exemple, d'un plan flou pour signifier le point de vue soit d'un personnage très faible, ivre, etc. (matérialité d'un corps), soit d'un personnage qui regarde par exemple à travers de l'eau (présence d'un œil). L'ocularisation interne primaire, plus sensorielle que syntaxique, aurait ainsi un évident potentiel à sensation comme point de vue narratif. Quant à la deuxième personne de la conjugaison iconique, que Jost n'identifie à aucun type d'ocularisation, on pourrait l'assimiler au regard à la caméra. Son nombre singulier ou pluriel relèverait, encore une fois, de la qualité simple ou complexe de l'objet de son interpellation, en l'occurrence le spectateur.

19 Pour le domaine sonore, Jost suit les mêmes critères et établit une nomenclature parallèle (les équivalences avec les personnes verbales restant donc inchangées). On parlerait d' auricularisation zéro pour une subjectivité sonore «soumise à la distance apparente du 
personnage ", c'est-à-dire pour une "perspective sonore " n'appartenant à aucun personnage (1989: 57). Ce serait le cas, par exemple, de la musique ou de la voix d'un narrateur qui n'est pas censée être entendue par les personnages, même si on l'associe à leurs actions. L'auricularisation interne secondaire, quant à elle, serait une première personne sonore « construite par le montage et le visuel » (Ibid.). À l'instar du raccord de regard, on pourrait affirmer ici que "n'importe quel son succédant à un plan mettant en scène un personnage qui écoute sera senti comme appartenant à son paysage sonore ». L'auricularisation interne primaire, enfin, consisterait dans une première personne marquée par "certaines déformations" qui «écartent la bande sonore des codes du réalisme » (Ibid.). Par exemple, un sifflement intense pour nous jeter dans la perspective d'un personnage à côté d'une explosion, ou l'absence totale de son pour nous faire sentir sa surdité, etc. L'auricularisation se montre ainsi, dans ses deux variantes internes, comme une perspective sonore ancrée dans l'image du personnage qui écoute, c'est-àdire comme une perspective sonore soumise à la perspective iconique.

La nomenclature de Jost nous permet ainsi de définir conjointement les perspectives iconiques et sonores qu'on pourrait adopter comme émetteur et/ou récepteur du récit. Au-delà de ses défauts comme ensemble (on n'a pas trouvé, par exemple, une première personne iconique du pluriel, et les deuxièmes personnes -iconiques et sonores- ne sont même pas mentionnées), c'est une nomenclature qui contribue de manière fondamentale au tournant iconique dans l'étude de la deixis. Face au concept verbal de personne, le concept d'ocularisation a la vertu de se référer à la perspective (au point de vue) au sens étroit de son étymologie: ce qu'on voit. Cette notion est conjuguée à sa première personne, comme il ne pouvait pas être autrement, par une mise-en-scène de l'acte luimême de regarder: principe fondateur de tout discours en images. L'association de l'ocularisation à la deixis personnelle nous permet ainsi de souligner la base iconique (sensible ou mentale) de toute tension pathétique comme adoption d'une perspective. Avec les deixis ad oculos et am Phantasma, l'ocularisation complète le tournant iconique du système déictique, qui avait ramené les notions d'espace, temps et personne au strict domaine du verbal.

\section{Partage d'un pathos}

21 À l'instar du concept de sensibilité de Keen, on distingue au pathos deux niveaux fondamentaux : les émotions (pathos psychique) et les sensations (pathos physique). Il y aurait également deux modalités du partage du pathos, telles qu'établies par la poétique classique. On pourrait, d'une part, partager le pathos d'un autre symbolique en tant que son semblable : ce serait l'empathie ou identification d'un « je sens avec toi » (Keen 2013 : 5). D'autre part, on pourrait aussi s'attacher "pathétiquement» (sensiblement) à cet autre symbolique sans s'identifier à lui : ce serait la sympathie d'un "je sens pour toi » (Ibid.). Dans les études littéraires occidentales, on a assimilé le degré superlatif de la sympathie narrative à la notion de catharsis telle que définie par Aristote à propos de la tragédie (1453b) : «le plaisir qui vient, à travers l'imitation, de la pitié et de la crainte » (1990: 124). La pitié serait le sentiment sympathique par excellence, en tant qu'on ne peut que la sentir pour quelqu'un (y inclus pour soi-même). La crainte, en revanche, pourrait être aussi bien empathique que sympathique, puisqu'on peut la sentir autant pour quelqu'un (y inclus pour soi-même) qu'avec quelqu'un. La tension pathétique passerait soit par l'empathie, soit par la sympathie, soit par un mélange des deux. Elle serait pourtant plus intense dans l'empathie, puisque celle-ci consiste dans une 
immersion totale (sentir avec), et non partielle (sentir pour). Par analogie à la tension chrono-topique, nous pourrions décrire maintenant la tension pathétique comme « un sentiment [total ou partiel] de sentir là ».

\section{Éthique du récit}

Le lien entre éthique et empathie, que nous avons pris ici comme prémisse, fait aujourd'hui l'objet d'un débat académique complexe dans les différents domaines qu'il traverse (narratologie, psychologie, philosophie, etc.). Quelques auteurs défendent ce lien comme nécessaire (Hogan 2001, 2010). D'autres vont jusqu'à affirmer que l'empathie narrative contribuerait à notre développement moral (Hoffman 2000), voire qu'elle créerait de bons citoyens du monde (Nussbaum 1990). Enfin, il y en a qui nient un lien univoque entre éthique et empathie, mais qui n'écartent pas la possibilité de les étudier corrélativement (Keen 2013: 14). Nous rejoindrons cette dernière position en faisant appel à l'hypothèse de Baron-Cohen, qui associe le concept du mal au manque d'empathie. Si discutable que l'hypothèse soit, elle a la vertu de suggérer une approche interactive (une tension) pour expliquer le sentiment qu'on aurait souvent, adoptant la perspective et partageant le pathos d'un autre, d'avoir affaire à un certain enjeu éthique.

D'après Baron-Cohen, ce que nous appelons couramment méchanceté (intention consciente de blesser quelqu'un) impliquerait par principe un manque d'empathie (2011: 156-160). Cependant, tous les manques d'empathie n'impliqueraient pas la méchanceté. Par exemple, les gens atteints du syndrome d'Asperger ont un degré zéro d'empathie qui est considéré comme éthiquement positif: «la manière dont leur cerveau traite l'information les amène paradoxalement à être super-moraux plutôt qu'immoraux ${ }^{16}$ " (Ibid.: 96). Ces individus essuient, pourtant, de grandes difficultés pour accéder à la dimension psychologique et éthique du récit : «Quand ils doivent lire de petites histoires pour porter des jugements sur les intentions, les motifs et l'état d'esprit d'un personnage, ou quand ils doivent lire le langage pour juger ce qu'une personne prétendait, ils montrent peu d'activité au cortex préfrontal médiodorsal ${ }^{17}$ » (Ibid. : 100-101), c'est-à-dire à la région du cerveau qui «devient active quand on pense aux idées et aux émotions d'autres gens (parfois appelé 'méta-représentation'), ainsi que quand on pense à nos propres idées et émotions ${ }^{18}$ » (Ibid. : 29). Sur ce constat empirique, nous entendrions que l'empathie narrative ne garantit pas en effet par principe une moralité définie chez le récepteur, mais qu'elle conditionne sa capacité à porter des jugements éthiques sur les personnages. C'est sur ce point que l'hypothèse de Baron-Cohen devient intéressante pour la narratologie. La notion du mal passant virtuellement par le manque d'empathie, la super-moralité d'Asperger se heurtant à ce même manque pour juger éthiquement les autres, ce serait juste à partir d'une certaine capacité de « sentir avec » qu'on atteindrait une dimension éthique à proprement parler, c'est-à-dire une articulation effective et autonome de la dichotomie bien/mal. Par extension, on pourrait dire que c'est en partageant (totale ou partiellement) un pathos qu'on accède au récit comme dimension éthique, c'est-à-dire que l'empathie et/ou la sympathie constituent, non pas le véhicule d'une moralité quelconque, mais la condition de possibilité pour une réception éthique du récit. 


\section{Récit musical}

24

$$
\begin{aligned}
& \text { les récits iconiques et verbaux, d'une part, et les récits musicaux, d'autre part. Ces } \\
& \text { derniers manqueraient de tension pathétique telle que nous l'avons définie ici. La } \\
& \text { musique ne dispose pas, en effet, d'un système articulant la perspective comme la deixis } \\
& \text { personnelle ou l'ocularisation. Rappelons, à ce sujet, que la perspective sonore de } \\
& \text { l'auricularisation décline les rapports image-son et qu'elle embrasse non seulement la } \\
& \text { musique, mais aussi la parole et le bruit. En absence d'une « deixis personnelle musicale ", } \\
& \text { c'est-à-dire d'un système nous permettant d'adopter un point de vue mélodique- } \\
& \text { harmonique se substituant à celui de notre réception, nous serions incapables de partager } \\
& \text { un pathos en termes d'empathie ou sympathie. Cela ne veut évidemment pas dire que la } \\
& \text { musique manque de dimension éthique ou sensible, mais que celle-ci ne se construit pas à } \\
& \text { travers une perspective personnelle conjuguée de manière systématique. Si nous parlions } \\
& \text { de musique et éthique, ce serait à propos d'unités culturelles dont la signification ne } \\
& \text { dépendrait pas de l'articulation conventionnelle d'un point de vue }{ }^{19} \text {. }
\end{aligned}
$$

\section{Tension télique}

On pourrait la définir comme l'immersion cognitive de l'émetteur et/ou le récepteur dans l'inconnue relative aux intentions d'un sujet, agent ou patient, vis-à-vis d'un fait qui n'a été pas encore accompli. C'est ce qu'on connaît plus couramment comme "suspense ». Si on appelle télique cette tension c'est parce que le concept d'intention implique une

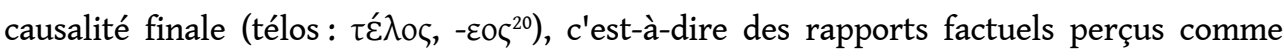
nécessaires en tant qu'intentionnés dans leur succession. La tension télique narrative met ainsi en rapport trois paramètres: les règles d'un monde représenté (Eco 1985b:35), l'ambiguïé relative de ces règles (hasard) et un libre arbitre se projetant au futur de ce monde représenté (intentions) (Eco ${ }^{21} 1985 a$ : 137).

D'un point de vue syntaxique, c'est ce qu'on a étudié sous le nom de proposition dépendante finale : l'énoncé qui, exprimant « un but» (afin que, pour, etc.), « suppose toujours une intention» (Wagner et Pinchon ${ }^{22} 1962$ : 587). D'un point de vue logique, il s'agit d'une causalité sensu lato ou même fallacieuse, en tant que non strictement nécessaire, dans la ligne du post hoc, ergo propter hoc décrié par la scolastique, qu'on pourrait compléter ici par un plus intentionnel ad hoc, ergo propter hoc, ou même par l'hybride intentionnel-chronologique ad hoc, ergo ante hoc. Substituant le terme motivation à celui d'intention, la narratologie structuraliste s'exprimait déjà dans ces termes (Genette 1969 : 94) : «La motivation est [...] l'apparence et l'alibi causaliste que se donne la détermination finaliste qui est la règle de la fiction ». Dans le même sens, des auteurs contemporains comme Jean-Michel Adam se réfèrent aux intentions des personnages comme un «voile de causalité » vis-à-vis de la structure logique du syllogisme (2005: 54). Dans sa propre nomenclature, la psychologie développementale abonde aujourd'hui dans un sens très semblable: "Lorsque la motivation (désir) est activée en une situation interpersonnelle, elle crée subjectivement une structure quasi narrative » (Stern $1995: 19)^{23}$.

L'intention s'avère ainsi le noyau de la tension télique du récit. Qu'elle soit explicite ou implicite, qu'on la mette en pratique ou non, qu'elle atteigne son but ou qu'elle le rate, 
l'intention détermine l'inconnue proprement narrative. Elle introduit la principale différence vis-à-vis d'autres types d'inconnue causale en tant qu'elle émane d'un autre fondement du récit: le pathos lié à une perspective. L'intention se mêle, en effet, de manière parfois inextricable aux émotions et aux sensations des personnages, nous encourageant elle aussi à l'empathie ou la sympathie. Comme le dit Keen : «les individus empathiques ont tendance à mieux comprendre les relations causales entre les événements racontés dans la fiction (Bourg) que ceux montrant un bas degré d'empathie ${ }^{24}$ » (2013: 10). L'opposition entre tension pathétique et tension télique reste, pourtant, pertinente dans la mesure où nous ne pouvons parler d'intention que lorsqu'une émotion ou une sensation se projette au futur.

\section{Suspense, curiosité, surprise}

28 En lignes générales, on pourrait apparenter le concept de tension télique au concept de suspense tel que défini par Baroni, qui le range parmi les modalités principales (avec la curiosité et la surprise) de son modèle général de tension (2007:254). Le suspense serait, d'après Baroni, le type de tension caractérisée par une "anticipation mêlée d'incertitude » qui se fonde « sur un pronostic de la situation narrative » (Ibid.). Revenant sur les travaux de M. Sternberg (1992) et de W. Brewer (1996), Baroni accorde au concept chronologique original une dimension cognitive (logique et thymique) dont il manquait. Même s'il ne mentionne pas le concept d'intention, celui-ci trouverait dans sa définition de suspense le cadre cognitif qui à notre avis lui est propre et qu'illustre par excellence la tension télique. Pour ainsi dire, l'intention (personnage) et le suspense (émetteur/ récepteur) seraient les deux faces d'une même monnaie (tension télique).

Nous entendrions, en vertu de cette réciprocité, que seul le suspense serait strictement indispensable à la tension télique narrative et donc à la tension narrative tout court. Certes, la curiosité et la surprise contribuent souvent aussi à la tension télique, parfois inextricablement mêlées au suspense. Elles n'auraient cependant pas la capacité de véhiculer par elles-mêmes l'indispensable élément intentionnel et, dans ce sens, elles ne sauraient être que des compléments (souvent très efficaces et complexes) de cette tension télique. Si nous considérons la curiosité et la surprise comme étant incapables de véhiculer une intention c'est parce qu'elles n'articulent pas, comme processus cognitifs, les deux éléments intentionnels fondamentaux : libre arbitre et orientation au futur.

\section{Tension télique musicale}

Développée à l'écart des tensions chrono-topique et pathétique, elle n'est pas associée à la figure d'un autre dont on partagerait le pathos dans l'espace-temps d'un monde représenté. On définirait ainsi la tension télique musicale comme l'immersion cognitive de l'émetteur et/ou le récepteur dans l'inconnue relative à l'évolution d'une série de faits mélodiques et/ou harmoniques et/ou rythmiques. C'est dans ce sens que nous parlerions d'un suspense musical. Si on appelle télique cette tension c'est parce que les rapports entre les faits musicaux sont perçus comme nécessaires en tant que motivés dans leur succession d'après certaines unités culturelles (par exemple, tonalité, mesure, etc.). Ces unités constituent les règles du «monde (musical) représenté » et interagissent avec nos hypothèses sur leur évolution. Il en va de même, bien entendu, pour les récits verbaux et iconiques, qui se construisent aussi sur des unités culturelles. Si l'influence de telles 
unités dans la tension télique devient plus évidente en musique, c'est parce qu'elles sont détachées des unités chrono-topiques et pathétiques, plus "visibles" narrativement (espace-temps, personnages) et donc capables de camoufler les unités téliques (intentions), relativement plus abstraites mais tout aussi déterminées culturellement.

31 L'absence de tension chrono-topique et pathétique nous permet ainsi de mieux isoler le processus cognitif du suspense en musique. On pourrait réduire celui-ci à l'interaction de nos souvenirs et de nos attentes vis-à-vis des unités culturelles que nous connaissons. Cette définition du suspense «dans l'abstrait» est, en fait, d'origine proprement musicologique. Elle est basée sur une idée de Theodor W. Adorno : « rien, en musique, ne reste isolé [...] chaque détail ne devient ce qu'il est que par le lien qui le rattache concrètement- à ce qui l'entoure et -par l'esprit- à ce qui est loin de lui : par le souvenir et l'attente» (1982: 7). Autrement dit, la syntaxe («ce qui l'entoure»), mais aussi une dimension supra-syntaxique («ce qui est loin de lui »), qui pourrait aller des différentes sections d'une même pièce jusqu'aux clichés stylistiques d'une époque, en passant par des principes harmoniques, etc., bref, par des unités culturelles. La réflexion d'Adorno nous permet de souligner, d'un point de vue cognitif, l'apparence causale du suspense aussi en musique : la confusion de ce qui vient souvent après (souvenir) et ce qui doit venir après (attente) du point de vue de l'émetteur et/ou le récepteur. Les suspenses verbal, iconique et musical partageraient ainsi une même logique du post hoc, ergo propter hoc déterminée par leurs respectives unités culturelles. C'est pourtant la musique qui nous permet de mieux relever la dynamique cognitive des souvenirs-attentes dans cette causalité sensu lato, qui s'avère ainsi un processus cognitif inter-linguistique large.

Le débat actuel en narratologie musicale, souvent penché sur la pertinence même du concept de récit en musique, pourrait à notre avis être abordé du point de vue de la tension télique. Les auteurs affirmant la pertinence du récit musical, comme Márta Grabócz, interprètent celui-ci comme le «mode d'organisation expressive d'une œuvre instrumentale » (2011:5), comme « les règles, les stratégies d'organisation des signifiés » et non pas comme « la volonté de voir une 'histoire racontée en musique' » (2009: 27). De la position contraire, ceux qui parlent plutôt de proto-récit, comme Jean-Jacques Nattiez, font allusion à un principe d'organisation très semblable : «Si l'on est tenté de parler de récit musical, c'est à cause [...] des effets de l'organisation syntaxique de la musique " (2011 : 3). Au-delà des étiquettes, on aurait l'impression qu'une partie du débat est basée sur l'allusion à une même notion (stratégies/effets d'organisation) par deux termes différents (récit, proto-récit). Aucune des deux positions associe la musique à ce qu'on a appelé ici tension chrono-topique ou pathétique. Leurs stratégies/effets d'organisation pourraient être expliqués, en revanche, par l'interaction de souvenirs et d'attentes culturellement déterminés qu'on a appelé ici tension télique. Ce serait spécialement clair dans le schéma de Grabócz, qui applique le concept de tension de Baroni à des unités culturelles musicales pour parler de suspense, introduisant ainsi une approche cognitive sur ses stratégies d'organisation (2011: 11-12). C'est en fait sur la base de la lecture de Grabócz que nous opposerions les récits verbaux et iconiques, d'une part, aux récits musicaux, d'autre part, en définissant les premiers par l'articulation de la triple tension narrative et les deuxièmes par une articulation particulière de la tension télique (suspense) seulement. Considérant qu'on ne peut pas trouver le concept de suspense ailleurs que dans le récit, nous serions enclins à garder une certaine association (l'étiquette étant secondaire) entre les notions de suspense musical et de récit ou narrativité. 


\section{Conclusions}

33 La sous-division du concept de tension de Baroni dans les versants chrono-topique, pathétique et télique nous a permis de développer nos deux hypothèses. D'une part, nous avons approfondi dans les aspects d'espace-temps, sensibilité et intention du récit d'un point de vue autant communicatif que cognitif. D'autre part, nous avons nuancé la frontière entre les récits verbaux-iconiques et les récits musicaux à l'intérieur de ce même système conceptuel. Pour la vérification textuelle de nos hypothèses nous renvoyons à l'analyse citée plus haut, qui nous semble représentative des problématiques ici posées ${ }^{25}$. Bien entendu, il faudrait ajouter à cette analyse beaucoup d'autres traversant les trois langages où la tension narrative est possible. Seulement de telles approches comparatistes pourraient à notre avis contribuer à définir l'objet d'étude narratologique comme acte communicatif et cognitif inter-linguistique.

34 Le besoin de nouvelles analyses, cependant, ne devrait pas nous empêcher de proposer des hypothèses cernant du moins provisoirement notre objet d'étude, c'est-à-dire essayant de répondre encore à la vieille question du récit. Nous en proposerons deux. La première hypothèse a été déjà avancée au long de ces pages. Revenant sur le concept de tension de Baroni, on pourrait suggérer que l'« attente d'un dénouement » à laquelle est « encouragé » le récepteur d'un récit iconique et/ou verbal passe par une triple tension: chrono-topique (immersion cognitive spatio-temporelle), pathétique (immersion cognitive sensible) et télique (immersion cognitive intentionnelle). Deux remarques s'imposent à propos de cette première hypothèse. D'une part, on voit comment la définition de Baroni, en faisant allusion à l'« attente d'un dénouement» et aux «traits passionnels » de la réception, mélange dans le seul concept de tension narrative ce que nous avons différencié comme tension télique et tension pathétique (tout en soulignant le lien intime, voire ambigu, entre les deux notions). La définition de Baroni omet ainsi seulement ce que nous avons appelé tension chrono-topique. D'autre part, le concept de tension a trouvé au long de ces pages quelques possibles synonymes. Nous l'avons identifié au concept d'immersion cognitive, mais on pourrait l'assimiler aussi au plus classique concept de fonction, étant donné leur commune nature relationnelle et constructiviste. Surtout si nous pensons au fonctionnalisme narratologique tel que défini aujourd'hui par des auteurs comme Sternberg: «Je suis un fonctionnaliste : je commence par demander quel est l'effet, et puis j'essaie de voir quelle(s) forme(s) peuvent déclencher cet effet ${ }^{26} »(2011: 40)$. On pourrait parler, de la sorte, d'une fonction narrative articulée sur une triple fonction: chrono-topique, pathétique et télique. Si nous avons préféré le terme de tension c'est parce qu'il souligne, dans son mélange métaphoriqueconceptuel, les éléments thymiques (pathétiques) du récit manquant dans le fonctionnalisme d'auteurs comme Sternberg.

Quant au récit musical, on pourrait affirmer que l'«attente d'un dénouement » à laquelle est « encouragé » l'émetteur et/ou le récepteur passe par une tension télique (suspense) mélodique et/ou harmonique et/ou rythmique. Il faut rappeler, pourtant, qu'on n'a pas développé à propos de la musique le critère pragmatique qu'on a ébauché pour parole et image (dynamique vs. non-dynamique), c'est-à-dire le critère touchant au plan physiquecognitif de l'émission et/ou de la réception des sons. L'approche pragmatique s'avère ainsi une importante lacune à combler dans l'étude du récit musical, ce qui appellerait une étude à part. La pragmatique musicale constitue en fait un domaine spécialement 
vaste et complexe, tenant compte que les quatre traits distinctifs du phénomène physique-harmonique (hauteur, intensité, timbre, durée) s'expliquent surtout comme articulations et effets physiques-cognitifs. Autrement dit, aux effets d'organisation (syntaxiques) du récit musical il faudrait ajouter ses effets perceptifs (pragmatiques).

Notre deuxième hypothèse fusionnerait les éléments précédents dans la vieille notion de récit, essayant d'opérer le même changement méthodologique que la tension mais de l'intérieur du vocabulaire traditionnel. De ce point de vue nous appellerions récit (iconique et/ou verbal) un acte communicatif et cognitif, fixé ou non sur un support, par lequel un récepteur et/ou un émetteur attend l'issue d'une série de faits et/ou d'actions liés à l'intention d'un sujet, agent ou patient, dont il partage la sensibilité dans des coordonnées spatiales, temporelles et personnelles définies et cohérentes. Par analogie, nous appellerions récit musical un acte communicatif et cognitif, fixé ou non sur un support, par lequel un récepteur et/ou un émetteur attend l'issue d'une série de faits mélodiques et/ou harmoniques et/ou rythmiques. La musique considérée, non comme partition ou comme lecture silencieuse de celle-ci, mais comme interprétation proprement sonore de hauteurs, timbres, intensités et durées mettrait encore en évidence le manque du critère pragmatique pour définir la signification du récit musical. Nos hypothèses se présentent ainsi comme faisant partie d'un travail en cours qui appelle, non seulement d'ultérieurs développements théoriques, mais aussi ces analyses textuelles qui les mettent à l'épreuve et qui leur accordent un plein sens.

\section{BIBLIOGRAPHY}

\section{Bibliographie}

Abbott, H. Porter. "Narrativity". In : Hühn, Peter et al. (eds.) : the living handbook of narratology. Hamburg : Hamburg University Press. URL = hup.sub.uni-hamburg.de/lhn/index.php? title=Narrativity\&oldid=1580 [view date : 14 Mar 2013]

Adam, Jean-Michel (2005 [1997]), Les textes : types et prototypes, Paris, Nathan.

Adorno, Theodor W. (1982 [1963]), Quasi una fantasia, Paris, Gallimard.

Aristote (1990), Poétique, Paris, Librairie générale française.

Baron-Cohen, Simon (2011), The Science of Evil. On Empathy and the Origins of Cruelty, New York, Basic Books.

Baroni, Raphaël (2007), La tension narrative. Suspense, curiosité et surprise, Paris, Seuil.

Begley, Sharon, « The Roots of Evil », Time (U.S. Edition), May 21, 2001.

Bell, Carl C. (1980), « Racism : a Symptom of the Narcissistic Personality Disorder », in Journal of the National Medical Association, Vol. 72, No. 7, 661-665.

Benjamin, Walter (2006 [1936]), L'œuvre d'art à l'époque de sa reproductibilité technique, Paris, Allia. 
Brewer, W., « The Nature of Narrative Suspense and the Problem of Rereading », in Vorderer, P., Wulff, H. et Friedrichsen, M. (dir.), Suspense, Conceptualizations, Theoretical Analyses, and Empirical Explorations, Mahwah, Lawrence Erlbaum Associates, 107-127.

Bühler, Karl (2009 [1934]), Théorie du langage, Marseille, Agone.

Burch, Noël (1991), La lucarne de l'infini, Paris, Nathan.

Cobussen, Marcel \& Nielsen, Nanette (2012), Music and Ethics, Franham, Ashgate.

Duchan, Judith F., et al. eds. (1995), Deixis in Narrative : A Cognitive Science Perspective, Hillsdale NJ, Lawrence Erlbaum.

Eco, Umberto (1972 [1968]), La structure absente, Paris, Mercure de France.

- (1985a [1979]), Lector in fabula, Paris, Grasset.

- (1985b [1983]), Apostille au Nom de la rose, Paris, Grasset.

Genette, Gérard (1969) : « Vraisemblable et motivation », Figures II, Paris, Seuil.

Grabócz, Márta (2009), Musique, narrativité, signification, Paris, L'Harmattan.

- (2011), « Métamorphoses de l'intrigue musicale (XIXe-XXe siècles) », Cahiers de Narratologie [en ligne], 21 / 2011, mis en ligne le 03 janvier 2012. URL : http://narratologie.revues.org/6503

Higgins, Kathleen (1991), The Music of Our Lives, Temple University Press.

Hoffman, Martin (2000), Empathy and Moral Development : Implications for Caring and Justice, Cambridge University Press.

Hogan, Patrick Colm (2001), « The Epilogue of Suffering : Heroism, Empathy, Ethics », SubStance $30,119-43$.

- (2010), What Literature Teaches us About Emotion, Cambridge University Press.

Jost, François (1989 [1987]), L'œil-caméra, Lyon, Presses Universitaires de Lyon.

Keen, Suzanne : "Narrative Empathy". In : Hühn, Peter et al. (eds.) : the living handbook of narratology. Hamburg ; Hamburg University Press. URL = hup.sub.uni-hamburg.de/lhn/ index.php?title=Narrative Empathy\&oldid=2044 [view date : 15 Mar 2013]

Meister, Jan Christoph : "Narratology". In : Hühn, Peter et al. (eds.) : the living handbook of narratology. Hamburg : Hamburg University Press. URL = hup.sub.uni-hamburg.de/lhn/ index.php?title=Narratology\&oldid=2050 [view date: 14 Mar 2013]

Nattiez, Jean-Jacques, « La Narrativisation de la musique », Cahiers de Narratologie [En ligne], 21 | 2011, mis en ligne le 21 décembre 2011, consulté le 22 novembre 2012. URL : http:// narratologie.revues.org/6467

Nünning, Ansgar (2003), « Narratology or Narratologies », in T. Kindt \& H.-H. Müller (eds). What Is Narratology? Questions and Answers Regarding the Status of a Theory, Berlin, de Gruyter, 239-75.

Pier, John \& Berthelot, Francis (2010), Narratologies contemporaines, Paris, Éditions des Archives contemporaines.

Preus, Anthony, Historical Dictionary of Ancient Greek Philosophy, The Scarecrow Press, Lanham, Maryland, 2007.

Staub, Ervin (1999), « The Roots of Evil : Social Conditions, Culture, Personality and Basic Human Needs », Personality and Social Psychology Review, Vol. 3, No. 3, Lawrence Erlbaum, 179-192. 
Stern, Daniel (1995), The Motherhood Constellation. A Unified View of Parent-Infant Psychotherapy, New York, Basic Books; trad. D. Cupa, La constellation maternelle, Paris, Calmann-Lévy, 1997.

Sternberg, M. (1992), « Telling in Time (II) : Chronology, Teleology, Narrativity », Poetics Today, no. $13,463-541$.

- (2011), « Reconceptualizing narratology. Arguments for a functionalist and a constructivist approach to narrative », Enthymema IV, 35-50.

Valles Calatrava, José R. (2008). Teoría de la narrativa. Una perspectiva sistemática. Madrid, Iberoamericana - Vervuert.

Wagner, R. L. et Pinchon, J., Grammaire du français classique et moderne, Paris, Hachette, 1962.

\section{NOTES}

1. Baroni critique, dans ce sens, à juste titre les travaux narratologiques de théoriciens de la réception comme Eco ou Wolfgang Iser : « Le principal reproche que nous pourrions adresser au traitement du suspense et de la curiosité par Eco et Iser tient à l'absence d'une réflexion portant sur les rapports qu'entretiennent ces deux formes d'incertitude anticipatrice dans le processus interprétatif avec les questions de la mise en intrigue et de la séquentialité du récit. C'est précisément sur ce rapport entre tension, intrigue et séquence narrative que nous allons nous pencher $[. .] ».(2007: 100)$.

2. Baroni considère, en effet, « complémentaires » des aspects cognitifs comme «l'identification et/ou la sympathie qu'est susceptible d'éprouver l'interprète à l'égard du protagoniste du récit » (2007 : 257). Ces aspects seront, en revanche, indispensables pour nous.

3. Une très bonne illustration du débat musicologique actuel autour des notions de récit/protorécit c'est le n²1 (2011) des Cahiers de Narratologie, coordonné par Raphaël Baroni et Alain Corbellari sous le titre "Rencontres de narratologies : perspectives sur l'intrigue musicale » ( http://narratologie.revues.org/6390).

4. Article à paraître (décembre 2014): "Trois langages pour la tension narrative: L'apprenti sorcier de Goethe, Dukas, Disney ", in Storytelling et tension narrative, Paris, L'Harmattan.

5. La notion de « chrono-topique » que nous emploierons ici ne recoupera pas dans ses critères le concept de chronotope tel que défini par Mikhail Bakhtine dans sa Théorie et esthétique du roman (1978), même si notre choix terminologique est évidemment basé sur celui de l'auteur russe.

6. Par "unité culturelle» nous entendons une "entité abstraite qui ne recouvre qu'une convention culturelle» (Eco 1972:63). Ces conventions culturelles seraient interculturelles dans la mesure où elles pourraient être traduites à d'autres langages (Ibid. : 64).

7. C'est justement le cas de L'apprenti sorcier d'Algar-Disney, que nous analysons dans l'article cité plus haut.

8. Le latin « oculos » se réfère, en effet, directement aux yeux et, dans la locution demonstratio ad oculos, il fait allusion au témoignage oculaire d'une réalité extra-verbale (référent) de nature implicitement iconique. L'allemand "phantasma", quant à lui, vient du grec classique « $\varphi \alpha ́ v \tau \alpha \sigma \mu \alpha$ » à travers le latin « phantasma » et équivaut au français «vision » (Petit Robert 2013). Ce dernier relèverait aussi, comme terme psychologique, du domaine iconique dans sa version in absentia : «Production de l'imagination par laquelle le moi cherche à échapper à l'emprise de la réalité » (Petit Robert 2013).

9. Nous empruntons ici l'opposition célèbre entre les concepts d'icône [signe analogique] et de symbole [signe non-analogique] de Charles Sanders Peirce à "Nomenclature and Divisions of Triadic Relations, as Far as They Are Determined", in Collected Papers, paragraphes 2.233-272. Nous ne reviendrons pourtant pas sur l'ensemble de la conception triadique peircienne du signe 
ni de la sémiosis, qui ne s'accordent pas aux concepts de mimesis ni de tension chrono-topique tel que nous les employons ici.

10. On interprétera ici le très polysémique terme pathos dans son sens plus général d'expérience (émotionnelle et sensorielle), d'après la définition du terme par Anthony Preus : "expérience, qu'elle soit bonne ou mauvaise » (« experience, either good or bad»). Preus considère, pourtant, le terme «émotions" ("feelings ») comme «dans beaucoup de cas [...] une très bonne traduction» de pathos (« in many cases [...] a very good translation») (2007: 196-197). Ma traduction.

11. «[...] our ability to identify what someone else is thinking or feeling and to respond to their thoughts and feelings with an appropriate emotion ». Ma traduction.

12. «[...] there are at least two stages in empathy : recognition and response ». Ma traduction.

13. Avant sa formulation par Baron-Cohen, on peut trouver ce lien hypothétique entre la notion du mal et le manque d'empathie chez plusieurs autres auteurs, comme Carl C. Bell dans ses études sur le rapport entre narcissisme et racisme (1980: 661-662), Ervin Staub dans ses analyses sur les conditionnements (sociaux, culturels, personnels, etc.) du mal (1999: 3), et bien d'autres. Le 21 mai 2001, sous le titre "The Roots of Evil», la revue Time (édition U.S.) faisait un bref compte rendu du congrès annuel de la Société Américaine de Psychiatrie, où plusieurs chercheurs posaient déjà l'idée que nous associons ici à Baron-Cohen (Begley 2001: 30). La formulation de ce dernier s'avérerait, dans ce sens, plus développée mais plus tardive aussi que celles de ses collègues.

14. «Many of the early studies of mind reading or empathy in people who are Zero-Positive relied on verbal tests (e.g., interpreting stories or sarcastic comments or labeling emotions) ». Ma traduction.

15. «[...] the sharing of feeling and perspective-taking induced by reading, viewing, hearing, or imagining narratives of another's situation and condition ». Ma traduction.

16. " [...] the way their brain processes information paradoxically leads them to be supermoral rather than immoral ». Ma traduction.

17. «When they have to read little stories to make judgments about a character's intentions, motives, and state of mind, or when they have to read language to judge what a person intended, they show reduced activity in the dorsomedial prefrontal cortex (AMPFC) ». Ma traduction.

18. «[...] is involved in thinking about other people's thought and feelings (sometimes called 'metarepresentation'), as well as when we think about our own thought and feelings ». Ma traduction.

19. On peut penser, par exemple, aux réflexions de Marcel Cobussen et Nanette Nielsen sur musique et éthique à propos du Wozzeck d'Alban Berg (Cobussen 2012). D'autres auteurs, comme Kathleen Higgins, sont allés jusqu'à considérer que l'écoute musicale stimule par elle-même nos capacités éthiques (Higgins 1991), un peu dans le même sens que Nussbaum accordait à la narrative littéraire la capacité de forger des bons citoyens du monde. Cette position nous paraît difficile à justifier.

20. Preus (2007: 259) : «Le télos c'est la fin ou le but d'une activité » (« The telos is the end or goal of some activity $)$. Ma traduction.

21. «Une narration est une description d'actions qui requiert pour chaque action décrite un agent, une intention de l'agent, un état ou monde possible, un changement, avec sa cause et le propos qui la détermine $[. .]$.$» .$

22. La définition classique exprime à la perfection l'ambiguïté causale de l'intention que nous attachons ici au récit : «But et conséquence sont des notions voisines. Elles se différencient en ce qu'un but suppose toujours une intention. Les locutions conjonctives qui servent à construire les propositions finales entraînent l'emploi du mode subjonctif dans la mesure où elles explicitent cette intention » (Ibid.).

23. Cité par Jean-Jacques Nattiez (2011: 33). 
24. Keen: «empathetic individuals tend to better grasp the causal relations between narrated events in fiction (Bourg) than those testing low in empathy ». Ma traduction.

25. Article à paraître (décembre 2014): "Trois langages pour la tension narrative : L'apprenti sorcier de Goethe, Dukas, Disney ", in Storytelling et tension narrative, Paris, L'Harmattan.

26. «I am a functionalist : I start by asking, What is the effect, and then I try to see what form(s) can trigger this effect ». Ma traduction.

\section{AUTHOR}

\section{GABRIEL SEVILLA}

Université de Genève 